\section{ALTERNATIVE SAFER APPROACH THROUGH THE CAROTID ARTERY FOR TRANSCATHETER AORTIC VALVE IMPLANTATION}

\section{To the Editor:}

We read with deep interest the case report by Modine and coauthors ${ }^{1}$ about the first transcatheter aortic valve implantation procedure through the left carotid artery access. The patient had a transient right hemiparesis after the procedure, probably because of the hypoperfusion of the left cerebral hemisphere despite the presence of a healthy circle of Willis or because of dissection of the left carotid artery.

We would like to mention a modification of the carotid access that addresses both complications. Direct visual introduction of a sheath through an open arteriotomy, then snaring the artery on the introducer and advancing the hydrophilic guidewire under fluoroscopic control would be helpful to prevent iatrogenic dissection. On the other hand, perfusion of the distal carotid artery with the aid of a centrifugal pump (eg, femoral artery to carotid artery) is an option to prevent the cerebral hypoperfusion. Our group successfully performed an experimental study for endovascular repair of the aortic arch with distal carotid perfusion. $^{2}$

Open carotid arteriotomy access with distal perfusion would also be helpful to prevent the embolic shower, at least to the ipsilateral cerebral hemisphere, during the instrumentation,

\footnotetext{
The Editor welcomes submissions for possible publication in the Letters to the Editor section that consist of commentary on an article published in the Journal or other relevant issues. Authors should: - Include no more than 500 words of text, three authors, and five references. - Type with double-spacing. - See http://jtcs.ctsnetjournals.org/misc/ifora.shtml for detailed submission instructions. $\bullet$ Submit the letter electronically via jtcvs.editorialmanager.com. Letters commenting on an article published in the JTCVS will be considered if they are received within 6 weeks of the time the article was published. Authors of the article being commented on will be given an opportunity of offer a timely response ( 2 weeks) to the letter. Authors of letters will be notified that the letter has been received. Unpublished letters cannot be returned.
}

balloon valvuloplasty, and implantation of the prosthesis. Allowing the free flow of the proximal carotid artery for a few seconds would clear off the debris.

We congratulate Modine and coauthors ${ }^{1}$ for their novel application of the transcatheter aortic valve implantation procedure.

Harun Arbatl, $M D^{a}$
Oguz Yilmaz, $M D^{b}$
Mustafa Cikrikcioglu, MD, PhD
${ }^{c}$ Department of Cardiovascular
Surgery
Maltepe University Faculty of
Medicine
Istanbul, Turkey
of Cardiovascular
Surgery
Department of
Istanbul, Turkey
${ }^{c}$ Division of Cardiovascular Surgery
Department of Surgery
University Hospital of Geneva
Geneva, Switzerland

\section{References}

1. Modine T, Lemesle G, Azzaoui R, Sudre A. Aortic valve implantation with the CoreValve ReValving System via left carotid artery access: first case report. J Thorac Cardiovasc Surg. 2010;140: 928-9.

2. Numan F, Arbatli H, Bruszewski W, Cikirikcioglu M. Total endovascular aortic arch reconstruction via fenestration in situ with cerebral circulatory support: an acute experimental study. Interact Cardiovasc Thorac Surg. 2008;7:535-8.

$$
\text { doi:10.1016/j.jtcvs.2010.10.052 }
$$

\section{Reply to the Editor:}

We congratulate Arbatlı and colleagues on their letter commenting on the carotid route used to achieve transcatheter aortic valve implantation. They give a reasonable explanation for the transient ischemic accident the patient experienced in our reported case. Indeed, hypoperfusion of the left cerebral hemisphere may have played a major role. This was probably mostly related to the carotid dissection that occurred initially. Arbatlı and colleagues suggest ameliorating this technique by direct visualization of sheath introduction through the open arteriotomy under fluoroscopic control. This precaution is now used in all our surgical transcatheter aortic valve implantations with carotid and subclavian arteries. ${ }^{1}$ They also suggest, on the basis of an experimental study that they performed, ${ }^{2}$ the use of distal perfusion centrifugal pumps. We do not recommend that technique. Indeed, the rationale behind transcatheter aortic valve implantation is to achieve minimally invasive implantation procedures. A complete study of circle of Willis perfusion, together with a full respect of the different steps to achieve this indication, could guarantee the success of this procedure. Indeed, without the occurrence of any complications, our group performed implantations in additional patients by this pathway. Finally Arbatl and colleagues point out the advantage of this surgical access as offering the possibility of preventing potential ipsilateral embolic shower during the balloon valvuloplasty and the valve implantation itself, which are the highest moments of cerebral embolic risks. Indeed, the carotid arteriotomy could be used as a window to clear off atherosclerotic debris during the procedure.

Transcatheter aortic valve implantation indications are growing. Exchanges of experiences are needed to optimize surgical implications and results.

Thomas Modine, MD

Georges Fayad, MD

Mohamad Koussa, MD

Service de Chirurgie

Cardiovasculaire

Hopital Cardiologique

Centre Hospitalier Régional et

Universitaire de Lille

Lille, France

\section{References}

1. Modine T, Obadia JF, Choukroun E, Rioufoul G Sudre A, Laborde JC, et al. Transcutaneous aortic valve implantation using the axillary/subclavian access: Feasibility and early clinical outcomes. J Thorac Cardiovasc Surg. Epub 2010 Apr 28. 
2. Numan F, Arbatli H, Bruszewski W, Cikirikcioglu M Total endovascular aortic arch. Reconstruction via fenestration in situ with cerebral circulatory support: an acute experimental study. Interact Cardiovasc Thorac Surg. 2008;7:535-8.

doi:10.1016/j.jtcvs.2010.11.038

\section{PALLIATIVE ARTERIAL SWITCH FOR TRANSPOSITION OF THE GREAT ARTERIES, VENTRICULAR SEPTAL DEFECT, AND PULMONARY VASCULAR OBSTRUCTIVE DISEASE}

\section{To the Editor:}

We read with interest the article by Lei and associates ${ }^{1}$ on palliative arterial switch for transposition of the great arteries, ventricular septal defect, and pulmonary vascular obstructive disease. We agree with them that the palliative arterial switch operation is definitely a better option than the palliative atrial switch operation. At our institution, we have performed 3 such operations in the past 2 years with a different approach. In these 3 patients, aged 1.5 years, 4.5 years, and 11 years, we used a simplified unidirectional-valved patch to close the ventricular septal defect (VSD). The surgical technique of construction of this patch was described in detail by us earlier. ${ }^{2}$ All 3 of these patients had severe pulmonary arterial hypertension with pulmonary artery systolic pressures of $110 \mathrm{~mm} \mathrm{Hg}, 126 \mathrm{~mm}$ $\mathrm{Hg}$, and $110 \mathrm{~mm} \mathrm{Hg}$ with a pulmonary vascular resistance of 9.5 units, 7 units, and 17.1 units, respectively. Intraoperative transesophageal echocardiography was used to see the direction of shunting across the patch after termination of cardiopulmonary bypass. In all 3 patients there was evidence of right-to-left shunt through the valved patch with mild systemic desaturation. Postoperative recovery was uneventful in all 3 patients except for episodic systemic desaturation in the first 2 patients, which was documented on echocardiography to be due to right-to-left shunt across the patch. The current follow-up of these patients is 3 months, 1 year, and 1 month, respectively, and these patients have systemic saturation in excess of $95 \%$ with no right-to-left shunting across the patch. The second patient had a cardiac catheterization 8 months after the operation, and the pulmonary artery systolic pressure has fallen to $49 \mathrm{~mm} \mathrm{Hg}$.

On the basis of our small experience with this technique, we believe that a unidirectional-valved patch may be a better alternative to an open or partially closed VSD. The obvious advantage is that a repeat invasive procedure to close the VSD is avoided. Moreover, this strategy also avoids the costs of a repeat cardiac catheterization and the substantial cost of the device used to close the VSD. In many parts of the world, these are important considerations.

Sachin Talwar, MCh Shiv Kumar Choudhary, MCh Balram Airan, MCh Cardiothoracic Centre All India Institute of Medical Sciences New Delhi, India

\section{References}

1. Lei B, Chen J, Cen J, Lui RC, Ding Y, Xu G, et al. Palliative arterial switch for transposition of the great arteries, ventricular septal defect, and pulmonary vascular obstructive disease: midterm outcomes. J Thorac Cardiovasc Surg. 2010;140:845-9.

2. Choudhary SK, Talwar S, Airan B. A simple technique of unidirectional valved patch for closure of septal defects. J Thorac Cardiovasc Surg. 2007; 134:1357-8.

doi:10.1016/j.jtcvs.2010.10.053

\section{Reply to the Editor:}

We read with interest the letter to the Editor by Talwar, Choudhary, and Airan on palliative arterial switch for transposition of the great arteries, ventricular septal defect, and pulmonary vascular obstructive disease. We appreciate the insightful contribution of their experience with unidirectional-valved patch to close the ventricular septal defect (VSD) in 3 patients, with apparently satisfactory short to midterm outcomes (mean follow-up of 5.3 months). The rationale for this approach is obviously an attempt to save the patients from the additional financial burden of future device closure of the VSD.

Although patients with VSDs presenting late for surgical closure with moderate-to-severe PVOD are rare in developed countries, they account for a significant percentage of our patient population in China (possibly the same in other developing countries), and we do have rather extensive experience with unidirectional-valved patch closures of VSDs in this unique patient population in the 1990s. What we found was that if there was no persistent or intermittent right-to-left shunt at the ventricular level, the unidirectional valve would be stuck and permanently closed within a relatively short period of time (a few weeks to a few months). In our article, $50 \%$ of our survivors (9/18) had persistent or even progressive PVOD up to 4 years of mean follow-up time. When these patients have occasional exacerbation of their pulmonary hypertension with suprasystemic right ventricular pressure, their VSD fenestrations become their only way of survival. With recent domestic production of the Amplatzer occluder device in China, the cost of transcatheter VSD device closure has fallen markedly. In our opinion, fenestrated VSD closure in this patient population confers a higher postoperative safety index with a small price to pay.

Jian Zhuang, $M D^{a}$ Raphael C. Lui, MD, FACS ${ }^{a}$ Bin-feng Lei, $M D^{b}$

${ }^{a}$ Department of Pediatric Cardiovascular Surgery Guangdong Provincial Cardiovascular Institute Guangdong General Hospital Guangzhou Guangdong, People's Republic of China

${ }^{b}$ Department of Cardiothoracic Surgery 